\title{
General Anesthesia for Dental Procedures in Children: A Comprehensive Review
}

\author{
Sunaakshi Puri ${ }^{1}$, Aditi Kapur ${ }^{2}$, Preethy J Mathew ${ }^{3}$
}

\begin{abstract}
With the advent of modern and safe anesthesia drugs and equipment, myriad of dental procedures are being safely conducted in children, including those with special needs. General anesthesia facilitates efficient intervention in children who would otherwise be uncooperative to dental interventions. However, providing general anesthesia for these procedures has its own risks and implications. Hence, a focused preanesthesia evaluation emphasizing upon the medical and behavioral comorbidities remains the cornerstone of providing safe anesthesia to these children. The fear of pain and anxiety due to parental separation are common in children which is managed by various pharmacological and non-pharmacological measures to enable a smooth transition onto the dental chair. Another important consideration in dental procedures is the sharing of airway by anesthesiologist and dentist. Airway protection with a definitive airway is therefore mandatory. Since children with special needs constitute a significant cohort undergoing dental procedures under general anesthesia, the anesthetic plan must be tailored for a smooth emergence as well.

We hereby discuss the various perioperative concerns in providing anesthesia to children for dental procedures emphasizing the preoperative and intraoperative implications and prerequisites to provide safe anesthesia.

Keywords: Dental procedures, General anesthesia, Pediatric.

Journal of Postgraduate Medicine, Education and Research (2022): 10.5005/jp-journals-10028-1555
\end{abstract}

\section{INTRODUCTION}

The origin of dental anesthesia dates back to 1844 , closely associated to the origin of modern anesthesia in 1848; when Horace Wells, a dentist by profession, performed painless dental extractions on patients after administering nitrous oxide. ${ }^{1}$ The advent of local anesthetics in 1905 revolutionized the practice of dentistry as dental interventions could be safely done under local anesthesia alone in awake patients. However, in children, local anesthesia alone is often not viable primarily due to their lack of cooperation. For minor procedures such as single tooth extraction or rehabilitation involving one or few teeth, local anesthesia may work with or without conscious sedation for the majority of children.

General anesthesia is required in a small and specific cohort of patients such as very young children less than three years of age who require complex dental treatment, those who do not allow procedures under conscious sedation, and those with special needs. Infants, children, and even adolescents with cognitive or psychological disabilities constitute a significant proportion who need general anesthesia for safe conduct of dental procedures.

\section{Overview}

\section{Advantages, Indications and Goals}

Administration of general anesthesia not only makes the procedure comfortable and safe for the children, but also efficient for the dental practitioner as extensive multi visit procedures can be performed without wasting effort and time to establish the child's cooperation in questionable cases and ending with multiple failed attempts adding to the distress of the child as well as the parents. It also saves the cost and inconvenience to the family, of multiple visits under conscious sedation which may be needed to complete the entire treatment. ${ }^{2}$ This enhanced efficiency facilitates extensive

\footnotetext{
1,3 Department of Anaesthesiology and Intensive Care, Postgraduate Institute of Medical Education and Research, Chandigarh, India

${ }^{2}$ Pedodontics and Preventive Dentistry Oral Health Sciences Center, Postgraduate Institute of Medical Education and Research, Chandigarh, India

Corresponding Author: Preethy J Mathew, Department of Anaesthesiology and Intensive Care, Postgraduate Institute of Medical Education and Research, Chandigarh, India, Phone: +91 9417800203, e-mail: tjpreethy@gmail.com

How to cite this article: Puri S, Kapur A, Mathew PJ. General Anesthesia for Dental Procedures in Children: A Comprehensive Review. J Postgrad Med Edu Res 2022;56(1):29-33.

Source of support: Nil

Conflict of interest: None
}

dental restorations in a single sitting while avoiding excessive amount of local anesthetic or sedative agents.

The following circumstances render definitive advantages in choosing general anesthesia over chair side behavior management or sedation for procedures:

- Lack of patient cooperation: The incidence of dental anxiety amongst preschoolers remains as high as $36.5 \% .^{3}$ Excessive anxiety and fear during dental visits are behavioral indicators to predict the need for general anesthesia. Moreover very young children are in the pre-cooperative stage and lack the ability to fully understand and comply with the complicated instructions required for complex oral rehabilitations. An attempt should always be made to develop a rapport with the young child chair-side prior to the procedure as they would routinely require preventive care such as fluoride application, and sometimes

(c) The Author(s). 2022 Open Access This article is distributed under the terms of the Creative Commons Attribution 4.0 International License (https://creativecommons. org/licenses/by-nc/4.0/), which permits unrestricted use, distribution, and non-commercial reproduction in any medium, provided you give appropriate credit to the original author(s) and the source, provide a link to the Creative Commons license, and indicate if changes were made. The Creative Commons Public Domain Dedication waiver (http://creativecommons.org/publicdomain/zero/1.0/) applies to the data made available in this article, unless otherwise stated. 
treatment of dislodged restorations, management of secondary caries, pain abscesses etc. at follow-up visits.

- Need for extensive dental restoration: Extensive dental restoration in children may result in higher cumulative doses of local anesthetic/sedative drugs due to repetitive administrations, whereas, general anesthesia offers a safer alternative.

- Contraindication to conscious sedation or local anesthesia: Adeno-tonsillar hypertrophy, syndromic facies, neurological deficit, mental retardation, and obesity confer risks during and after administration of pharmacological sedation. General anesthesia in expert hands is deemed safer than conscious sedation. Previous history of allergy to local anesthetic is another situation when general anesthesia may be considered.

- Presence of acute orofacial infection: The acidic environment in the inflamed tissues remarkably reduces the efficacy of local anesthetics as the nondiffusible charged form of local anesthetics with poor membrane permeability predominate in the acidic $\mathrm{pH}$.

The spectrum of pediatric dental conditions/pathologies requiring general anesthesia for procedural interventions are listed in Table 1.

However, administering general anesthesia is not without its inherent risks. Therefore, the anesthesiologist must strike a balance between the risks and benefits to individualize the plan of anesthesia as per the nature of procedure, requirement of surgeon, and the condition of the child. The complexity of dental procedure, medical status and cognitive development of the child, and the adequacy of available resources and manpower, together determine the type of anesthesia technique suitable for the child. The guiding principles for administration of general anesthesia in pediatric dental setting are indicated in Figure 1.

\section{Providing Anesthesia in the Dental Suite: Outside the Comfort Zone of the Operation Theater}

Providing anesthesia in an unfamiliar location outside the operating room has always been challenging for anesthesiologists. Dental suite is one such location where the routine facilities of an operation theater may not be available. For example, in operating rooms, general anesthesia is administered to patients on a standard

Table 1: Dental procedures that may require general anesthesia in children 1. Odontogenic infections.

2. Severe early childhood caries requiring extensive rehabilitation.

3. Tooth extractions: erupted, unerupted, impacted, supernumerary tooth.

4. Frenulum attachments: ankyloglossia, maxillary frenula.

5. Oral pathologies:

Mucosal conditions such as mucocele,

Reactive or inflammatory lesions,

Neoplastic lesions.

6. Trauma: Fracture mandible for dental wiring or fixation.

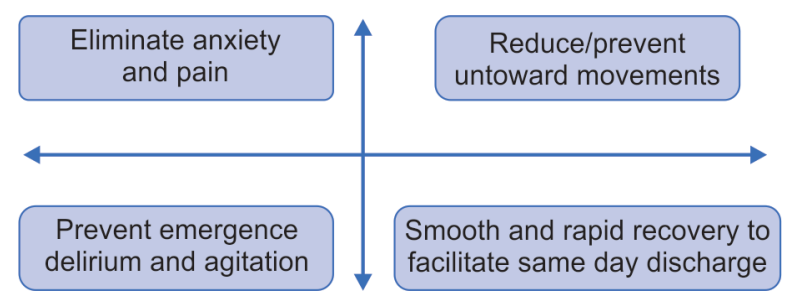

Fig. 1: Anesthetic goals in pediatric dental procedures operating table which has features to rapidly tilt the patient's body toward head, feet or sides, whenever required. On the contrary, a dental chair lacks these features posing difficulties during anesthetic procedures. Similarly, operation theatres usually have well-established preoperative room and postanesthesia care units with patient monitoring facilities and trained nursing personnel which may not be available in the dental suite. Hence, a collaborative effort by the dental and anesthesiology departments is imperative to ensure patient safety during anesthetic care in dental suite. The American Society of Anesthesiology (ASA) has drafted standard guidelines for establishing a Non-Operating Room Anesthesia location. ${ }^{4}$ Diligent adherence to these guidelines will go a long way to provide quality care. The essential components of equipment check for Non-Operating Room Anesthesia are described by the mnemonic SOAP-ME and include the following:

\begin{tabular}{ll}
\hline S Suction & $\begin{array}{l}\text { A functioning suction apparatus and age } \\
\text { appropriate suction catheters. } \\
\text { R Oxygen }\end{array}$ \\
Reliable oxygen source, with at least one spare \\
oxygen cylinder. \\
Age appropriate airway equipment. \\
P Pharmacy & $\begin{array}{l}\text { Basic anesthetic drugs and their antagonist; } \\
\text { emergency drugs. }\end{array}$ \\
M Monitors & $\begin{array}{l}\text { Standard ASA monitors- electrocardiography, } \\
\text { blood pressure, pulse oximetry, end-tidal carbon } \\
\text { dioxide, and temperature. }\end{array}$ \\
E Equipment & $\begin{array}{l}\text { e.g., defibrillator. } \\
\text { (special) }\end{array}$
\end{tabular}

\section{Anesthetic Management}

\section{Preanesthetic Evaluation and Advice}

A focused comprehensive evaluation of the child by a trained anesthesiologist is essential for all children whereas those with co-morbid illnesses, neuropsychiatric disorders, or syndromes should be evaluated well before the day of procedure. Such preanesthetic evaluation will facilitate appropriate planning of preoperative and postoperative periods and thus, prevent undue cancellations or delays on the day of the procedure. Details regarding the previous anesthesia exposure, if any, can provide useful information such as nature of anesthesia, drugs administered, airway device employed, and history of nausea and vomiting. Apart from the routine medical history and systemic examination, three aspects need special mention in evaluation for pediatric dental anesthesia, viz. behavioral issues, medical comorbidities and airway examination.

- Behavioral issues: Children are known to be vulnerable to develop caries in the early years of life due to limited understanding of caregivers about the implications of oral hygiene. Associated developmental and/or psychological disability makes them further prone to dental caries. The common developmental disorders encountered in pediatric dental practice that influences preanesthetic preparation are discussed here:

- Autism: Autism spectrum disorder is a heterogeneous developmental disorder, characterized by deficits in communication and social interaction, culminating in significant impairment in normal functioning. These traits, along with the unacceptance to the unfamiliar environment of a dental clinic, exposure to light, noise, and unusual taste 
makes it near impossible to separate the child from the parent for induction of anesthesia. Enquiring about the normal routine, likes, dislikes, and comforting tools and techniques for these children can help the anesthesiologist plan the process of induction and extubation. ${ }^{5}$

- Down syndrome: Children with Down's syndrome have delayed development and usually have a large tongue, hypertrophied adenoids, and tonsils, making them potential candidates for obstructive sleep apnea and difficult airway. A detailed airway examination will help to decide the most suitable airway plan. Since congenital heart disease is not uncommon, preoperative evaluation may include an echocardiography to assess any cardiovascular risk. Given the high incidence of gastroesophageal reflux disease (GERD), antiaspiration prophylaxis is warranted in these children. ${ }^{6}$

- Cerebral palsy:Cerebral palsy is a group of neurodevelopmental disorders affecting the muscle tone, motor function, movement, and posture of the child. Spasticity and seizures constitute the major neurological manifestations. It is important to enquire about ongoing anticonvulsant and antispasmodic medications and to continue these perioperatively. GERD and aspiration are also common. Antireflux medications must therefore be continued. Perioperative chest physiotherapy will help those with poor cough, poor secretion clearance, and recurrent chest infections. However, the possibility of postoperative ventilation must be explained in patients with poor respiratory function. ${ }^{7}$

- Medical comorbidities: Certain medical illnesses are associated with higher incidence of dental caries. Preanesthetic evaluation should focus on detection and optimization of these.

- Cardiac anomalies: Children with congenital heart disease (CHD) have poorer oral health, significantly more enamel defects, positional anomalies and crowding as compared to other children. ${ }^{8}$ In addition, multiple hospital admissions and visits tend to make them more anxious. The plan of anesthesia should be based upon the type of anomaly (cyanotic/acyanotic, repaired/unrepaired/partially repaired) and the physiological status of the child. It is extremely crucial to administer antibiotic prophylaxis to children with CHD who are prone to infective endocarditis ${ }^{8}$ (Table 2). Risk of infective endocarditis is highest in dental procedures such as tooth extraction or abscess drainage, which involve manipulation of gingival tissue or periapical region of teeth, or mucosal breach. The antibiotic regimen includes Ampicillin $50 \mathrm{mg} / \mathrm{kg} \mathrm{i} / \mathrm{v}$, or cefazolin or ceftriaxone $50 \mathrm{mg} / \mathrm{kg} \mathrm{i} / \mathrm{v}$. For children allergic to ampicillin, alternatives include cephalexin $50 \mathrm{mg} / \mathrm{kg}$ or azithromycin $15 \mathrm{mg} / \mathrm{kg}$ or doxycycline $4.4 \mathrm{mg} / \mathrm{kg}$. ${ }^{9}$ The antibiotic must be administered 30-60 minutes before surgical incision.

- Epilepsy: Epileptic seizures may lead to dental trauma and subsequent need for intervention. Continuation of antiepileptic medications in the perioperative period is essential. Avoidance of anesthetic drugs with potential proconvulsant properties; and identification and treatment of dyselectrolytemia, hypoglycaemia, and other treatable causes of seizure during the perioperative period will minimize the risk. The enzyme inhibiting effect of antiepileptics may alter the metabolism of anesthetic drugs. Hence, the dosages of relevant anesthetic drugs should be altered accordingly.
Table 2: Indications for IE prophylaxis

Previous history of IE.

Unrepaired congenital cyanotic heart diseases including palliative procedures.

Repaired congenital heart defects with residual defects or prosthetic material or device.

- Gastroesophageal reflux disease (GERD): Reflux of acidic contents contribute to poor oral hygiene and development of dental caries. The antireflux medications must be continued perioperatively in these children. Organic causes of reflux such as hiatal hernia also put the child at risk of aspiration. Need for rapid sequence induction and intubation in these children should be assessed in the preanesthetic evaluation.

- Airway examination: The most consistent and noteworthy characteristic of dental surgery is sharing airway with the surgeon. Meticulous preoperative assessment of airway by the anesthesiologist ensures adequate preparation and subsequent airway management. Special emphasis is to be laid upon evaluation of nasal passages as nasal intubation is often preferred over the oral route to secure the airway.

Since dental pathologies are often encountered in children with various syndromes, their airway management can be potentially difficult, necessitating advanced airway equipment, and expertise of an experienced anesthesiologist. Age and weight appropriate advanced airway equipment as well as expert help may not be readily available in the dental suite. Hence, children with anticipated difficult airway should be scheduled in operation theater for the smooth conduct of procedure.

Prior to general anesthesia, it is essential to fast the children as per nil per os (NPO) guidelines to minimize the risk of regurgitation and pulmonary aspiration. Delivering the correct NPO guidelines will avoid delays due to inadequate fasting, and prevent patient discomfort due to excessively prolonged fasting. For children scheduled for day care procedures, the dentist needs to provide verbal as well as written fasting instruction in the clinic. The recent NPO guidelines comprise of fasting to solids and liquids for 6 hours, breast milk for 4 hours, and clear fluids for 2 hours. ${ }^{10}$

\section{Premedication and Parental Separation}

Fear of pain, anxiety due to parental separation, irritability due to hunger and lack of understanding make children apprehensive, and uncooperative before the procedure. Such problems are further magnified in children with neurodevelopmental problems. Various pharmacological and nonpharmacological methods can be utilized to alleviate anxiety and thus facilitate parental separation.

- Pharmacological measures: Drugs with sedative, anxiolytic, and amnestic effects are best suited to facilitate smooth parental separation and inhalational induction. Midazolam, a short acting benzodiazepine remains the most popular agent for uncooperative children without any comorbid illness. The highly selective alpha- 2 agonist, dexmedetomidine, acts as a central sympatholytic agent, and induces a natural sleep-like state. Recently introduced into the practice of dentistry, it does not cause respiratory depression, making it suitable in children. It also contributes to reduction in emergence delirium, without delaying patient recovery and discharge. Ketamine, an NMDA receptor antagonist, is considered superior for use in children 
with congenital heart disease due to its relative cardiovascular stability and minimal effect on the respiratory system.

- Nonpharmacological measures: Interventions such as parental or caregiver's presence during induction of anesthesia, quiet environment, use of distraction techniques such as interactive video games or allowing the child to hold his/ her personal toys are some of the measures to facilitate smooth induction process while avoiding restraint. These techniques play an important role in children at risk of respiratory depression with pharmacological agents, syndromic children with potential difficult airway and in children with behavioral traits such as aggression, which makes administration of premedication difficult. These are also useful in autistic children in whom the effect of premedication may be unpredictable.

\section{Conduct of Anesthesia}

\section{Induction of Anesthesia}

Induction is usually carried out via inhalation route, typically using sevoflurane. Alternatively, intravenous route can be used depending on easy placement of intravenous cannula. Rapid induction due to low blood gas solubility, lack of pungency, and sweet odor make sevoflurane the agent of choice for inhalation induction. The most common drug available for intravenous induction is propofol. Mixing $10 \mathrm{mg}$ lignocaine per $100 \mathrm{mg}$ of propofol, mini Bier's block with lignocaine through the indwelling cannula, injecting propofol slowly through a running $\mathrm{i} / \mathrm{v}$ line are some techniques that may reduce the local pain caused by propofol, rendering IV induction more comfortable for the patient. The dose of propofol may be higher in children on antiepileptics due to hepatic enzyme induction and in patients with CHD comprising of left to right shunts, both resulting in lower effect-site concentration. Ketamine is the preferred agent for induction in children with cyanotic congenital heart diseases. Tracheal intubation may require administration of muscle relaxant. However, it may be omitted if highly potent opioids such as remifentanil are available. Intraoperative monitoring includes the five standard ASA monitors such as continuous electrocardiography, pulse oximetry, non-invasive blood pressure, end-tidal carbon dioxide concentration, and temperature.

\section{Airway Management}

The main considerations with dental surgery are the shared airway and the presence of blood and debris in the oral cavity, with possible risk of aspiration. Airway protection with a definitive airway device is therefore necessary. Endotracheal intubation is the most suitable and safe means to secure the airway. Some anesthesiologists prefer a nasotracheal placement to keep the endotracheal tube away from the surgical field, facilitating repair by providing unobstructed access to all four quadrants of mouth, with minimal to no interference with ventilation. It also facilitates assessment of tooth alignment and occlusion. Nasal mucosa and nasopharynx are highly vascular, necessitating adequate vasoconstriction and decongestion prior to intubation. The commonly used agents include xylometazoline nasal drops $0.05 \%$ (for infants and small children) and $0.1 \%$ (for older children), phenylephrine $0.25 \%$ and adrenaline. These agents, when applied with cotton swabs or pledgets, cause vasoconstriction within 15-20 minutes. Orotracheal intubation can be considered if there is a contraindication to nasal intubation (such as suspicion of large adenoids, cleft palate repair, deviated nasal septum etc.), unavailability of suitable nasal preparation drugs, or lack of experience with nasal intubation.
Amongst the various endotracheal tubes available, the preformed oral/nasal Ring-Adair-Elwyn tube and the flexometallic tube are the most suitable. The risk of intraoperative kinking of tube and the consequent interference with ventilation is minimal with these devices. Water proof dressings for tube fixation is recommended to prevent accidental inward or outward movement of the tube. Extensive bilateral restoration may require the anesthesiologist to reposition an oral endotracheal tube from one angle of mouth to the other during the procedure. Micro aspirations cannot be ruled out even when using a cuffed ETT. Therefore, following tracheal intubation, a throat pack is usually inserted to protect the airway from soiling. Confirmation of removal of pack is extremely vital before extubation to avoid laryngospasm, airway obstruction, and other untoward complications of a retained throat pack.

An alternative to endotracheal intubation is to use a wire-reinforced version of the classic laryngeal mask airway (LMA), known as the flexible LMA. The reinforced tubing provides more versatility to this device, allowing better access. Using an LMA allows the anesthesiologist to maintain spontaneous ventilation throughout the surgery, making it a suitable choice for children with neuromuscular diseases, who have an increased sensitivity to nondepolarizing muscle relaxants.

\section{Maintenance of Anesthesia}

Most dental procedures are performed on an ambulatory basis. Dental procedures carried out under general anesthesia tend be somewhat overtreated to avoid chances of second visit failures. Thus extractions are preferred over pulpectomies for abscessed teeth and pulpectomies are given preference over pulpotomies for pulp with questionable diagnosis. At the same time another significant factor to be considered is overall treatment duration which may have a direct correlation with emergence time and duration of postanesthetic recovery. The plan of anesthesia must therefore aim to facilitate early recovery and discharge. In children with cerebral palsy, the anesthetic requirement has been estimated to be lower. ${ }^{11,12}$ There is resistance to nondepolarizing muscle relaxants, attributable to drug interaction with anticonvulsants, and chronic immobilization. Care must be taken while positioning to prevent pressure sores, especially during prolonged surgeries. Also these children are more prone to develop hypothermia perioperatively. ${ }^{7}$ It is a common practice in oral surgery to instil or infiltrate the surgical field with dilute adrenaline solution or adrenaline soaked gauzes. It is the responsibility of the anesthesiologist to ensure use of adequate concentration and volume of adrenaline in order to avoid the adverse effect of arrhythmia from excessive adrenaline use. Simultaneous use of volatile anesthetics particularly halothane increases the chances of arrhythmia with adrenaline. Stimulation of the trigeminal nerve during dental work may also contribute to intraoperative arrhythmias. Majority of dental surgeries are not associated with severe postoperative pain. Short acting opioids such as fentanyl, remifentanil along with NSAIDS, or acetaminophen usually suffice. Coadministration of local anesthetic agent further reduces the anesthetic requirement and contributes to analgesia.

\section{Emergence}

Prior to initiating awakening from anesthesia, the anesthesiologist must ensure removal of throat pack and gauzes from the oral cavity. A thorough and gentle oropharyngeal suctioning, often aided by positioning the patient slightly head down, ensures a clear airway. Following reversal of neuromuscular blockade, awake extubation should be carried out after return of consciousness and 
muscle power. Return of airway reflexes is important to facilitate a safe extubation.

\section{Postoperative Management}

The postoperative period is often complicated by emergence delirium and postoperative nausea and vomiting. Dual antiemetic prophylaxis is therefore warranted. 5HT3 antagonist such as $0.15 \mathrm{mg} / \mathrm{kg}$ of ondansetron, along with $0.1-0.2 \mathrm{mg} / \mathrm{kg}$ dexamethasone significantly reduces the risk of postoperative nausea and vomiting as compared to individual therapy alone.

Children with autism and other behavioral disorders react poorly to new environment, therefore emergence agitation is fairly common. Hence measures to prevent it should be considered. Involving parents in the recovery phase, providing a quiet environment, and early removal of intravenous catheters facilitate smooth recovery. A bolus dose of dexmedetomidine $(0.5-1 \mathrm{mcg} / \mathrm{kg})$ given at the end of surgery may be used both as a preventive and therapeutic measure. ${ }^{5}$ Triple therapy comprising of dexmedetomidine, midazolam and antiemetic have also been shown to reduce emergence delirium the most. ${ }^{13}$ Early discharge serves best for these children, therefore the plan of anesthesia should fulfil the criteria of ambulatory surgery, favoring same day discharge.

\section{Conclusion}

With the increasing number of children requiring dental procedures under general anesthesia, it is imperative to have a thorough understanding of the various aspects specific to dental anesthesia, keeping the risk vs benefit ratio in view. A holistic approach, consisting of thorough preoperative assessment, formulating an adequate anesthetic plan and avoiding as well as managing common complications associated with dental surgeries in both normal and cognitively impaired children, will ensure safe administration of dental procedures under general anesthesia. While majority of surgeries can be conducted on an outpatient basis, it is of utmost importance to screen patients requiring admission.

\section{References}

1. Hewitt PB. Anesthesia for dental and orofacial surgery. Healy TEJ, Knight PR (ed). Wylie and Churchill-Davidson's A Practice of Anesthesia, Boca Raton, FL, CRC Press, 2003;829-36.
2. Kapur A, Jain K, Goyal A, et al. Oral midazolam sedation for uncooperative children in outpatient paedodontics: time for reappraisal. SAAD Dig 2016;32:14-16.

3. Grisolia BM, Santos AP, Dhyppolito IM, et al. Prevalence of dental anxiety in children and adolescents globally: a systematic review and metanalysis. Int J Pedtr Dent 2020; 31(2):168-183. DOI: 10.1111/ ipd. 12712

4. American Society of Anesthesiologists (ASA), Statement on Nonoperating Room Anesthetizing Location. 2018. Available from: https://www.asahq.org/standards-and-guidelines/statementon-nonoperating-room-anesthetizing-locations. [Accessed October 20].

5. Wittkugel EP, Samol NB. Special Pediatric Disorders. In: Davis PJ, Cladis FP (ed). Smith's Anesthesia for Infants and Children, 9th edition. St. Louis, MO, Elsevier, 2017;1210-1219.

6. Oliveira MB, Machado HS. Perioperative management of patients with Down Syndrome: a review. J Anesth Clin Res 2018;9(4):1-14. DOI: $10.4172 / 2155-6148.1000816$

7. Wang $\mathrm{YC}$, Lin $\mathrm{IH}$, Huang $\mathrm{CH}$, et al. Dental anesthesia for patients with special needs. Acta Anaesthesiol Taiwan 2012;50(3):122-125. DOI: 10.1016/j.aat.2012.08.009

8. Hughes $S$, Balmer R, Moffat M, et al. The dental management of children with congenital heart disease following the publication of Paediatric Congenital Heart Disease Standards and Specifications. Br Dent J 2019;226(6):447-452. DOI: 10.1038/s41415-019-0094-0

9. Wilson WR, Gewitz M, Lockhart PB, et al. Prevention of Viridans Group Streptococcal Infective Endocarditis: a scientific statement from the American Heart Association. Circulation 2021;143:e963-e978. DOI:10.1161/CIR.0000000000000969.

10. Practice guidelines for preoperative fasting and the use of pharmacologic agents to reduce the risk of pulmonary aspiration: application to healthy patients undergoing elective procedures: an updated report by the American Society of Anesthesiologists Task Force on preoperative fasting and the use of pharmacologic agents to reduce the risk of pulmonary aspiration. Anesthesiology 2017;126:376-393. DOI: 10.1097/ALN.0000000000001452

11. Saricaoglu F, Celebi N, Celik M, et al. The evaluation of propofol dosage for anesthesia induction in children with cerebral palsy with bispectral index (BIS) monitoring. Paediatr Anaesth 2005;15(12): 1048-1052. DOI: 10.1111/j.1460-9592.2005.01658.x

12. Frei FJ, Haemmerle MH, Brunner R, et al. Minimum alveolar concentration for halothane in children with cerebral palsy and severe mental retardation. Anaesthesia 1997;52(11):1056-1060. DOI: 10.1111/j.1365-2044.1997.257-az0376.x

13. Wang HY, Chen TY, Li DJ, et al. Association of pharmacological prophylaxis with the risk of pediatric emergence delirium after sevoflurane anesthesia: an updated network meta-analysis. J Clin Anesth 2021;75:110488. DOI:10.1016/j.jclinane.2021.110488. Epub ahead of print. PMID: 34481361. 